\title{
Local conditions for global representations of quadratic forms
}

\author{
by \\ RAiner Schulze-Pillot (Saarbrücken)
}

In [5] Ellenberg and Venkatesh prove that for any integral positive definite quadratic form $f$ in $n$ variables there is a constant $C(f)$ such that integral quadratic forms $g$ of square free discriminant in $m \leq n-5$ variables with minimum $\mu(g)>C(f)$ are represented by $f$ if and only if they are represented by $f$ locally everywhere, i.e., over $\mathbb{R}$ and over all $p$-adic integers. If one fixes an odd prime $p$ not dividing the discriminant of $f$ one can find a constant $C^{\prime}(f, p)$ such that representability is even guaranteed for $g$ of rank $m \leq n-3$ with $\mu(g)>C^{\prime}(f, p)$, provided the discriminant of $g$ is further restricted to be prime to $p$.

It is mentioned in [5] that I have suggested to replace the condition of square free discriminant on $g$ by a weaker condition. This suggestion is worked out here. Combining our version of the result of [5] with results of Kitaoka we also obtain some new cases in which with a suitable fixed prime $q$ the only condition on $g$ (apart from $\mu(g)>C(f, q)$ and representability of $g$ by $f$ locally everywhere) is bounded divisibility of the discriminant of $g$ by $q$. Moreover, results on extensions of representations as given in [1, 2] can be obtained with new dimension bounds. We take the occasion to reformulate some of the proofs of [5] in a way that is closer to other work on the subject.

We will work throughout in the language of lattices as described e.g. in $[12,15]$ (with the exception of Theorem 11). We fix a totally real number field $F$ with ring of integers $\mathfrak{o}$ and a totally positive definite quadratic space $(V, Q)$ over $F$ of dimension $n \geq 3$; the quadratic form $Q$ may be written as $Q(x)=\langle x, x\rangle$ with a scalar product $\langle$,$\rangle on V$. We denote by $O_{V}(F)$ the group of isometries of $V$ with respect to $Q$ (the orthogonal group of the quadratic space $(V, Q))$, by $O_{V}(\mathbb{A})$ its adelization, and by $S O_{V}(F)$ resp. $S O_{V}(\mathbb{A})$ their subgroups of elements of determinant 1 . For a lattice $\Lambda$ on $V$ we denote its automorphism group (or unit group) $\left\{\sigma \in O_{V}(F) \mid \sigma(\Lambda)=\Lambda\right\}$

2000 Mathematics Subject Classification: Primary 11E12; Secondary 11E04.

Key words and phrases: representation of quadratic forms, integral quadratic forms. 
by $O_{\Lambda}(\mathfrak{o})$, and similarly for the local or adelic analogues. The minimum of $\Lambda$ is

$$
\mu(\Lambda):=\min \left\{N_{\mathbb{Q}}^{F}(Q(\mathbf{x})) \mid \mathbf{x} \in \Lambda, \mathbf{x} \neq \mathbf{0}\right\} .
$$

For the question which lattices have large minimum it does not matter whether we choose this definition or $\min \left\{\operatorname{Tr}_{\mathbb{Q}}^{F}(Q(\mathbf{x})) \mid \mathbf{x} \in \Lambda, \mathbf{x} \neq \mathbf{0}\right\}$ instead (see the remark in [7, p. 139]).

Definition 1. Let $\tilde{F}$ denote either $F$ or one of its completions $F_{w}$ at a finite place $w$ of $F$, and $\tilde{\mathfrak{o}}$ either $\mathfrak{o}$ or its completion $\mathfrak{o}_{w}$ at the same $w$; analogously we write $\tilde{V}$ for $V$ or its completion $V_{w}$ at $w$. Let $\Lambda$ be an $\tilde{\mathfrak{o}}$-lattice of full rank $n=\operatorname{dim}_{\tilde{F}}(\tilde{V})$ on $\tilde{V}$, and $M$ an $\tilde{\mathfrak{o}}$-lattice of rank $m$ on a quadratic space $\left(\tilde{W}^{\prime}, Q^{\prime}\right)$ of dimension $m$ over $\tilde{F}$.

The lattice $M$ is represented by $\Lambda$ if there is an $\tilde{F}$-linear embedding $\varphi: \tilde{W}^{\prime} \rightarrow \tilde{V}$ with $Q(\varphi(w))=Q^{\prime}(w)$ for all $w \in \tilde{W}^{\prime}$ (the embedding is isometric) for which $\varphi(M) \subseteq \Lambda$.

If $M$ is represented by $\Lambda$ through the isometric embedding $\varphi$ we put $\tilde{W}:=\varphi\left(\tilde{W}^{\prime}\right)$ and say that the representation $\varphi$ is primitive if $\tilde{W} \cap \Lambda=\varphi(M)$.

The representation $\varphi$ of $M$ by $\Lambda$ has imprimitivity bounded by $c \in \tilde{\mathfrak{o}}$ if $c v \in \varphi(M)$ for all $v \in \tilde{W} \cap \Lambda$. In particular, for $c=1$ a representation with imprimitivity bounded by 1 is the same as a primitive representation.

We say that the o-lattice $M$ on the $F$-space $W^{\prime}$ is represented (resp. primitively represented, resp. represented with imprimitivity bounded by $c \in \mathfrak{o}$ ) by the o-lattice $\Lambda$ on the $F$-space $V$ locally everywhere if the completion $M_{w}$ is represented (resp. primitively represented, resp. represented with imprimitivity bounded by $c$ ) by the completion $\Lambda_{w}$ for all (finite or infinite) places $w$ of $F$.

Remark. Two representations $\varphi: M \rightarrow \Lambda, \psi: M \rightarrow \Lambda^{\prime}$ by lattices $\Lambda, \Lambda^{\prime}$ on $V$ of the o-lattice $M$ are in the same genus of representations if they are in the same $O_{V}\left(\mathbb{A}_{F}\right)$-orbit, i.e., if for every place $w$ of $F$ one has $\varrho_{w} \in O_{V}\left(F_{w}\right)$ with $\varrho_{w}\left(\Lambda_{w}\right)=\Lambda_{w}^{\prime}$ and $\psi_{w}=\varrho_{w} \varphi_{w}$ (where $\varphi_{w}, \psi_{w}$ are the extensions of $\varphi, \psi$ to isometries from $M_{w}=\mathfrak{o}_{w} M$ to $\Lambda_{w}=\mathfrak{o}_{w} \Lambda$, resp. $\left.\Lambda_{w}^{\prime}=\mathfrak{o}_{w} \Lambda^{\prime}\right)$. Obviously two representations in the same representation genus have the same primitivity/imprimitivity properties.

In our setting the given local representations determine a genus of representations of $M$ by lattices in the genus of $\Lambda$, and all representations of $M$ that we are going to construct will lie in that genus of representations.

As in [5] we have an action of the adelic orthogonal group $O_{V}(\mathbb{A})$ on the set of lattices on $V$; the isometry classes of lattices in the orbit of $\Lambda$ under this action are (by the Hasse-Minkowski theorem) precisely the isometry classes of lattices in the genus gen $(\Lambda)$ of $\Lambda$. The isometry classes of lattices in the orbit of the subgroup $O_{V}^{\prime}(\mathbb{A})$ of adelic transformations of determinant 
and spinor norm 1 (or equivalently under the adelic version $\operatorname{Spin}_{V}(\mathbb{A})$ of the spin group of $V$ ) are the classes in the spinor genus (or spin genus) $\operatorname{spn}(\Lambda)$ of $\Lambda$. As usual we write $\theta(\varphi)$ for the spinor norm of a special orthogonal transformation $\varphi$.

LEMma 2. Let $\Lambda$ be an o-lattice of rank $n$ on $V$ and $M$ an o-lattice on $W^{\prime}$ of rank $m \leq n-3$, and assume that $M$ is represented by $\Lambda$ locally everywhere with imprimitivity bounded by the number $c \in \mathfrak{o}$. Then $M$ is (globally) represented with imprimitivity bounded by c by some lattice $\Lambda^{\prime}$ in the spinor genus of $\Lambda$.

Proof. The same statement without the condition on bounded imprimitivity is proved in $[13,6]$. The proof here is essentially the same.

The Hasse-Minkowski theorem allows us to assume $M \subseteq W^{\prime} \subseteq V$, and hence the set $T$ of places $w$ of $F$ for which $M_{w} \nsubseteq \Lambda_{w}$ is finite and consists only of nonarchimedean places. By adding the finitely many places $w$ to $T$ for which $M_{w}$ is not primitive in $\Lambda_{w}$ we can even assume that $M_{w}$ is a primitive sublattice of $\Lambda_{w}$ for all $w \notin T$.

By assumption, for all $w \in T$ we have isometric embeddings $\varphi_{w}$ : $W^{\prime} \rightarrow V$ such that $\varphi_{w}\left(M_{w}\right) \subseteq \Lambda_{w}$ and $c \cdot \Lambda_{w} \cap \varphi_{w}\left(W_{w}^{\prime}\right) \subseteq \varphi_{w}\left(M_{w}\right)$, and by Witt's theorem we can assume the $\varphi_{w}$ to be elements of the orthogonal group $O_{V}\left(F_{w}\right)$; it is easy to see that we can assume them to have determinant 1 . Since the orthogonal complement $U_{w}$ of $\varphi_{w}\left(W_{w}^{\prime}\right)$ in $V_{w}$ has dimension $\geq 3$ its special orthogonal group contains transformations of arbitrary spinor norm (see [15]). Choosing a transformation $\psi_{w} \in S O_{U_{w}}\left(F_{w}\right)$ with $\theta\left(\psi_{w}\right)=\theta\left(\varphi_{w}\right)$ and extending $\psi_{w}$ to $V_{w}$ by letting it act as identity on $\varphi_{w}\left(W_{w}^{\prime}\right)$ we obtain $\varrho_{w}=\psi_{w} \circ \varphi_{w} \in S O_{V}\left(F_{w}\right)$ with $\theta\left(\varrho_{w}\right)=1$ and with $\varrho_{w}\left(M_{w}\right) \subseteq \Lambda_{w}$ and $c \cdot \Lambda_{w} \cap \varphi_{w}\left(W_{w}^{\prime}\right) \subseteq \varrho_{w}\left(M_{w}\right)$. The lattice $\Lambda^{\prime}$ on $V$ defined by $\Lambda_{w}^{\prime}=\Lambda_{w}$ for $w \notin T$ and $\Lambda_{w}^{\prime}=\varrho_{w}^{-1} \Lambda_{w}$ for $w \in T$ is then in the spinor genus of $\Lambda$ and contains $M$ with imprimitivity bounded by $c$.

REMARK. (i) The above argument allows us to include congruence conditions on the representation of $M$ by $\Lambda^{\prime}$ : If $S$ is a finite set of nonarchimedean places of $F$, and representations $\sigma_{v}: M_{v} \rightarrow \Lambda_{v}$ are fixed for all $v \in S$, one can choose for any $s \in \mathbb{N}$ the representation $\varrho: M \rightarrow \Lambda^{\prime}$ in such a way that

$$
\varrho(m)-\sigma_{v}(m) \in p^{s} \Lambda_{v}^{\prime} \quad \text { for all } v \in S
$$

and all $m \in M$. This is proved as above, adding the places of $S$ to the finite set $T$ of places chosen above.

(ii) If the quadratic space $(V, Q)$ is not totally definite but indefinite at at least one archimedean place of $F$, the strong approximation theorem for the spin group [3] implies that the spinor genera of lattices on $V$ coincide with the isometry classes. In that case the question of global representations 
is therefore completely settled by the above lemma (see $[4,13,6]$ ), and the only case needing further consideration is the totally definite case.

For definite $(V, Q)$ we can use (following Eichler and Kneser) the strong approximation theorem with respect to some finite place $w$ of $F$ for which the completion $V_{w}=V \otimes F_{w}$ is isotropic, and extend the lattices on $V$ to "arithmetically indefinite" lattices over the larger ring $\mathfrak{o}^{(w)}=F \cap F_{w} \prod_{v \neq w} \mathfrak{o}_{w}$; over this ring spinor genera and isometry classes coincide again, and we obtain (similarly to [7]):

LEMMA 3. With notations and assumptions as in Lemma 2 let $w$ be a finite place of $F$ for which the completion $V_{w}=V \otimes F_{w}$ is isotropic. Then every isometry class in the spinor genus of $\Lambda$ has a representative $\tilde{\Lambda} \subseteq V$ with $\tilde{\Lambda}_{v}=\Lambda_{v}$ for all finite places $v \neq w$ of $F$ and $\tilde{\Lambda}_{w} \in \operatorname{Spin}_{V}\left(F_{w}\right) \Lambda_{w}$. Furthermore, there is a lattice $\Lambda^{\prime \prime}$ in the spinor genus of $\Lambda$ with $\Lambda_{v}^{\prime \prime}=\Lambda_{v}$ for all finite places $v \neq w$ of $F$ and $\Lambda_{w}^{\prime \prime} \in \operatorname{Spin}_{V}\left(F_{w}\right) \Lambda_{w}$ such that $M$ is represented by $\Lambda^{\prime \prime}$ with imprimitivity bounded by $c$.

Proof. To prove the second assertion we take first a lattice $\Lambda^{\prime}$ in the spinor genus of $\Lambda$ as guaranteed by Lemma 2, and may assume that $M$ is a sublattice of $\Lambda^{\prime}$ with imprimitivity bounded by $c$. We write $\Lambda^{\prime}=\varphi \Lambda$ with an adele $\varphi=\left(\varphi_{v}\right) \in \operatorname{Spin}_{V}\left(\mathbb{A}_{F}\right)$. The strong approximation theorem allows us to write $\varphi=\sigma \psi$ with $\sigma \in \operatorname{Spin}_{V}(F)$ and $\psi=\left(\psi_{v}\right) \in \operatorname{Spin}_{V}\left(\mathbb{A}_{F}\right)$ with $\psi_{v} \Lambda_{v}=\Lambda_{v}$ for all finite $v \neq w$. Setting $\Lambda^{\prime \prime}=\sigma^{-1} \Lambda^{\prime}=\psi \Lambda$ we have $\Lambda_{v}^{\prime \prime}=\Lambda_{v}$ for all finite places $v \neq w$ of $F$, and $\Lambda^{\prime \prime}$ contains the lattice $\sigma^{-1}(M)$, which is isometric to $M$, with imprimitivity bounded by $c$. The same argument shows the first assertion of the lemma.

REMARK. Again we may include congruence conditions at finitely many nonarchimedean places $v \neq w$.

Lemma 4. Let $w$ be a nonarchimedean place of $F$, and $\Lambda_{w}$ be an $\mathfrak{o}_{w^{-}}$ lattice of rank $n$ on $V_{w}=V \otimes F_{w}$. Let $\mathcal{W}$ be a set of regular subspaces of $V_{w}$, put $M_{W}:=W \cap \Lambda_{w}$ for $W \in \mathcal{W}$ and assume that the (additive) $w$-adic valuation $\operatorname{ord}_{w}\left(\operatorname{disc}\left(M_{W}\right)\right)$ of the discriminants of the $M_{W}$ is bounded by some $j \in \mathbb{N}$ independent of $W$. Then the set $\mathcal{W}$ is contained in the union of finitely many orbits under the action of the compact open subgroup

$$
\tilde{K}_{w}:=\operatorname{Spin}_{\Lambda_{w}}\left(\mathfrak{o}_{w}\right)=\left\{\tau \in \operatorname{Spin}_{V}\left(F_{w}\right) \mid \tau\left(\Lambda_{w}\right)=\Lambda_{w}\right\}
$$

of $\operatorname{Spin}_{V}\left(F_{w}\right)$.

Proof. This is stated and proved (with $\operatorname{Spin}_{\Lambda_{w}}\left(\mathfrak{o}_{w}\right)$ replaced by some unspecified compact subset of $\left.\operatorname{Spin}_{V}\left(F_{w}\right)\right)$ as Lemma 2 in [5]. We show here that it is an immediate consequence of known facts from the local theory of quadratic forms. 
The boundedness condition on $\operatorname{ord}_{w}\left(\operatorname{disc}\left(M_{W}\right)\right)$ leaves only finitely many possibilities for the Jordan decomposition of $M_{W}$ and hence for the isometry class of $M_{W}$. It is well known (see Satz 30.2 of [14]; the proof given there for lattices over $\mathbb{Z}$ applies to $\mathfrak{o}_{w}$-lattices as well) that for a given $M$ there are only finitely many $O_{\Lambda_{w}}\left(\mathfrak{o}_{w}\right)$-orbits of representations of $M$ by $\Lambda_{w}$, in other words, only finitely many orbits of sublattices of $\Lambda_{w}$ which are isometric to $M$. Obviously, each of these orbits breaks up into finitely many orbits under the action of $\operatorname{Spin}_{\Lambda_{w}}\left(\mathfrak{o}_{w}\right)$.

Proposition 5. Let $V / F$ be as before and denote as before by $\tilde{K}_{v}$ the compact open subgroup $\operatorname{Spin}_{\Lambda_{v}}\left(\mathfrak{o}_{v}\right)=\left\{\tau \in \operatorname{Spin}_{V}\left(F_{v}\right) \mid \tau\left(\Lambda_{v}\right)=\Lambda_{v}\right\}$ for each finite place $v$ of $F$. Let $w$ be a fixed finite place of $F$, and $T_{w}$ a regular isotropic subspace of $V_{w}=V \otimes F_{w}$ with $\operatorname{dim}\left(T_{w}\right) \geq 3$. Let $G_{w}=\operatorname{Spin}_{V}\left(F_{w}\right)$, $H_{w}=\operatorname{Spin}_{T_{w}}\left(F_{w}\right)$ and

$$
\Gamma:=\operatorname{Spin}_{V}(F) \cap \operatorname{Spin}_{V}\left(F_{w}\right) \prod_{v \neq w} \tilde{K}_{v} .
$$

Let $\left(W_{i}\right)_{i \in \mathbb{N}}$ be a sequence of subspaces of $V$ (over the global field $F$ ) such that $\left(W_{i}\right)_{w}^{\perp}=\xi_{i} T_{w}$ for each $i$, with the elements $\xi_{i}$ from a fixed compact subset of $G_{w}$. If no infinite subsequence of the $W_{i}$ has a nonzero intersection, then the sets $\Gamma \backslash \Gamma \xi_{i} H_{w}$ are becoming dense in $\Gamma \backslash G_{w}$ as $i \rightarrow \infty$, i.e., for every open subset $U$ of $G_{w}$ one has $U \cap \Gamma \xi_{i} H_{w} \neq \emptyset$ for sufficiently large $i$.

Proof. This is stated as Claim in 2.5 (p. 269) of [5]; it is proven there using results of Ratner and Margulis/Tomanov from ergodic theory.

Proposition 6. Let $V / F, Q$ be as before and $\Lambda$ a lattice of full rank $n$ on $V$; moreover, let $w$ be a fixed finite place of $F$. Let $\left(W_{i}\right)_{i \in \mathbb{N}}$ be a sequence of regular subspaces $W_{i}$ of $V$ (over the global field $F$ ) of dimension $m \leq \operatorname{dim}(V)-3$ with the property that no infinite subsequence of the $W_{i}$ has a nonzero intersection and the orthogonal complement of $\left(W_{i}\right)_{w}$ in $V_{w}$ is isotropic. Assume moreover that the w-adic additive valuations $\operatorname{ord}_{w}\left(\operatorname{disc}\left(\left(W_{i}\right)_{w} \cap \Lambda_{w}\right)\right)$ remain bounded by some fixed integer $j$ as $i$ varies. Then $\tilde{M}_{i}=W_{i} \cap \Lambda$ is primitively represented by all lattices in the spinor genus $\operatorname{spn}(\Lambda)$ for sufficiently large $i$.

Proof. This is stated and proven in [5] as Proposition 2 with $j=1$. The proof does not change for arbitrary $j$; for the reader's convenience we go through it and reformulate it slightly.

As before we put $\tilde{K}_{v}=\operatorname{Spin}_{\Lambda_{v}}\left(\mathfrak{o}_{v}\right)$ for all finite places $v$ of $F$ and

$$
\Gamma:=\operatorname{Spin}_{V}(F) \cap \operatorname{Spin}_{V}\left(F_{w}\right) \prod_{v \neq w} \tilde{K}_{v} .
$$

By Lemma 4 the $\left(\tilde{M}_{i}\right)_{w}$ (and hence the $\left(W_{i}\right)_{w}$ ) fall into finitely many orbits under the action of the compact open group $\tilde{K}_{w}$; by treating the orbits 
separately we may therefore assume that they all belong to the same orbit, i.e., with $T_{w}=\left(W_{1}\right)_{w}^{\perp}$ we have $\left(W_{i}\right)_{w}^{\perp}=\xi_{i} T_{w}$ with $\xi_{i} \in \tilde{K}_{w}$ for all $i$.

Given an arbitrary isometry class in the spinor genus of $\Lambda$ we use Lemma 3 to obtain a representative $\tilde{\Lambda} \subseteq V$ of that isometry class with $\tilde{\Lambda}_{v}=\Lambda_{v}$ for all finite places $v \neq w$ of $F$ and $\tilde{\Lambda}_{w}=g_{w} \Lambda_{w}$ for some $g_{w} \in G_{w}$.

By Proposition 5 for every open set $U \subseteq G_{w}$ there is an $i_{0}$ such that we have $U \cap \Gamma \xi_{i} H_{w} \neq \emptyset$ for $i \geq i_{0}$.

Taking

$$
U=g_{w} \tilde{K}_{w} \subseteq G_{w}
$$

we obtain $i_{0}$ such that for all $i \geq i_{0}$ one has elements $\gamma_{i} \in \Gamma, \eta_{i} \in H_{w}$, $\kappa_{i} \in \tilde{K}_{w}$ with $g_{w} \kappa_{i}=\gamma_{i} \xi_{i} \eta_{i}$. The lattice $\Lambda_{i}^{\prime}:=\gamma_{i}^{-1} \tilde{\Lambda}$ is then in the isometry class of $\tilde{\Lambda}$; it satisfies $\left(\Lambda_{i}^{\prime}\right)_{v}=\Lambda_{v}$ for all finite $v \neq w$ and

$$
\left(\Lambda_{i}^{\prime}\right)_{w}=\gamma_{i}^{-1} g_{w} \Lambda_{w}=\xi_{i} \eta_{i} \Lambda_{w}=\xi_{i} \eta_{i} \xi_{i}^{-1} \Lambda_{w} .
$$

From this and $\left.\xi_{i} \eta_{i} \xi_{i}^{-1}\right|_{\left(W_{i}\right)_{w}}=\operatorname{Id}_{\left(W_{i}\right)_{w}}$ we see $\tilde{M}_{i}=W_{i} \cap \Lambda_{i}^{\prime}$, i.e., we have the required primitive representation by a lattice in the given isometry class.

Proposition 7. Let $(V, Q), \Lambda$ be as before, let $\left(M_{i}\right)_{i \in \mathbb{N}}$ be a sequence of $\mathfrak{o}$-lattices of rank $m \leq n-3$ and assume that with some fixed $c \in \mathfrak{o}, j \in \mathbb{N}$ and some fixed finite place $w$ of $F$ one has:

(i) $M_{i}$ is represented locally everywhere by $\Lambda$ with imprimitivity bounded by $c$ and with isotropic orthogonal complement at $w$ for all $i$.

(ii) $\operatorname{ord}_{w}\left(\operatorname{disc}\left(\left(M_{i}\right)_{w}\right)\right) \leq j$ for all $i$.

(iii) The sequence $\left(\mu\left(M_{i}\right)\right)_{i \in \mathbb{N}}$ of the minima of the $M_{i}$ tends to infinity. Then there is an $i_{0} \in \mathbb{N}$ such that $M_{i}$ is represented (with imprimitivity bounded by $c$ ) by all isometry classes in the genus of $\Lambda$ for all $i \geq i_{0}$.

Proof. By Lemma 2 we may concentrate on lattices in the spinor genus of $\Lambda$ and assume that all $M_{i}$ are represented by $\Lambda$ with imprimitivity bounded by $c$; we may also replace the $M_{i}$ by their images under those representations and assume $M_{i} \subseteq \Lambda$. We denote by $W_{i}$ the $F$-space generated by $M_{i}$ and assume that there is an infinite subsequence of the $W_{i}$ with nonzero intersection.

If $\mathbf{x} \neq \mathbf{0}$ is a vector in this intersection we may assume (replacing it by a suitable multiple) that $\mathbf{x} \in \Lambda$ and hence $\mathbf{x} \in W_{i} \cap \Lambda$ for infinitely many $i$. But then the condition of bounded imprimitivity of the representations of the $M_{i}$ implies $c \mathbf{x} \in M_{i}$ for infinitely many $i$, i.e., we have a vector of length $N_{\mathbb{Q}}^{F}\left(c^{2} Q(\mathbf{x})\right)$ in infinitely many of the $M_{i}$, which contradicts the assumption (iii) that the minima of the $M_{i}$ tend to infinity.

We have shown that no infinite subsequence of the $W_{i}$ has a nonzero intersection, so by Proposition 6 there is an $i_{0}$ such that $W_{i} \cap \Lambda$ is primitively 
represented by all lattices in the spinor genus of $\Lambda$ for all $i \geq i_{0}$, which implies the assertion about the $M_{i}$.

Theorem 8. Let $(V, Q), \Lambda$ be as before, fix a finite place $w$ of $F$ and $j \in \mathbb{N}, c \in \mathfrak{o}$. Then there exists a constant $C:=C(\Lambda, j, w, c)$ such that $\Lambda$ represents all $\mathfrak{o}$-lattices $M$ of rank $m \leq n-3$ satisfying:

(i) $M$ is represented by $\Lambda$ locally everywhere with imprimitivity bounded by $c$ and with isotropic orthogonal complement at the place $w$.

(ii) $\operatorname{ord}_{w}\left(\operatorname{disc}\left(M_{w}\right)\right) \leq j$.

(iii) The minimum of $M$ is $\geq C$.

The representation may be taken to have imprimitivity bounded by $c$. The isotropy condition is satisfied automatically if $m \leq n-5$ or if $\operatorname{disc}\left(\Lambda_{w}\right)$ and $\operatorname{disc}\left(M_{w}\right)$ are units at $w$.

Proof. If the assertion were wrong we could take a sequence of lattices $M_{i}$ of rank $m$ satisfying (i) and (ii) above and in addition $\mu\left(M_{i}\right) \geq i$ such that no $M_{i}$ is represented by all classes in the genus of $\Lambda$. This would contradict the previous proposition.

The final remark about the isotropy condition follows easily from known facts about quadratic spaces over nonarchimedean local fields.

REMARK. (i) All arguments used above remain valid if one imposes additional congruence conditions at finite places outside the fixed place $w$ on the representations, starting out from the versions with congruence conditions of Lemma 2 and Lemma 3 as indicated in the remarks after those lemmata and incorporating the congruence conditions into the choice of the compact open groups $\tilde{K}_{v}$ for the $v \neq w$.

(ii) The theorem without the condition on $\operatorname{ord}_{w}(\operatorname{disc}(M))$ and without the condition on bounded imprimitivity has been proven in [7] by a different method for $n \geq 2 m+3$.

We note that for $n \geq 2 m+3$ it is an easy consequence of the local theory of lattices that there is a $c \in \mathfrak{o}$ such that a lattice of rank $m$ which is represented locally everywhere by $\Lambda$ is in fact represented locally everywhere by $\Lambda$ with imprimitivity bounded by $c$ (see e.g. [8, Theorem 2]), so that (without using [7]) we can omit the condition of bounded imprimitivity of the local representations if $n \geq 2 m+3$. More generally the same is true if the Witt index of $V$ is at least $m$.

(iii) From the point of view of the analytic theory the condition of bounded imprimitivity for the local representations is natural: If one splits the number $r(\Lambda, M)$ of representations of $M$ by $\Lambda$ into the sum of a main term given by Siegel's weighted average $r(\operatorname{gen}(\Lambda), M)$ and an error term, a calculation of the local densities shows that the main term grows only slowly 
(or not at all) if $M$ varies in a sequence of lattices represented locally only with growing imprimitivity (see for example [12, Theorem 5.6.5 c)]).

The usual method of showing that the main term grows faster in absolute value than the error term so that the sum must eventually become positive may therefore not work if one considers such a sequence, and it even appears plausible that the small average number of representations may be due to the fact that not all isometry classes do represent the lattices considered.

(iv) For applications it would sometimes be desirable to have a different condition than growing minimum of the lattices to be represented, e.g. growing discriminant plus representability of all successive minima. It appears that at least the present method is not able to give such a result: If we consider an infinite sequence of lattices $M_{i} \subseteq \Lambda$ whose minimum is bounded, there must be infinite subsequences having a nonzero intersection, since there are only finitely many vectors of given length in $\Lambda$. Propositions 5 and 6 cannot therefore be applied to such a situation. One can, however, easily adapt the method to the case of extensions of representations of some fixed lattice as treated in $[1,2]$ (see Corollary 9 below).

(v) At least presently there seems to be no way to obtain an effective bound on the constant $C$ of the theorem. The result is therefore probably more useful in the form of Proposition 7. We note that for $n \geq 2 m+3$ the method of [7] does at least in principle allow one to find an effective bound.

Corollary 9. Let $(V, Q), \Lambda$ be as before, and fix a finite place $w$ of $F$ and $j \in \mathbb{N}, c \in \mathfrak{o}$. Let $R \subseteq \Lambda$ be a fixed $\mathfrak{o}$-lattice of rank $r$, let $\sigma: R \rightarrow \Lambda$ be a representation of $R$ by $\Lambda$, and assume that $R_{w}$ is unimodular. Then there exists a constant $C:=C(\Lambda, R, j, w, c)$ such that if $M \supseteq R$ is an o-lattice of rank $m \leq n-3$ and:

(i) for each place $v$ of $F$ there is a representation $\tau_{v}: M_{v} \rightarrow \Lambda_{v}$ with $\left.\tau_{v}\right|_{R_{v}}=\sigma_{v}$ with imprimitivity bounded by $c$ and with isotropic orthogonal complement in $\Lambda$ at the place $w$,

(ii) $\operatorname{ord}_{w}\left(\operatorname{disc}\left(M_{w}\right)\right) \leq j$,

(iii) the minimum of $M \cap(F R)^{\perp}$ is $\geq C$,

then there exists a representation $\tau: M \rightarrow \Lambda$ with $\left.\tau\right|_{R}=\sigma$. The representation may be taken to have imprimitivity bounded by $c$. The isotropy condition is satisfied automatically if $m \leq n-5$ or if $\operatorname{disc}\left(\Lambda_{w}\right)$ and $\operatorname{disc}\left(M_{w}\right)$ are units at $w$.

Proof. This is proven in the same way as Theorem $\mathrm{IV}^{\prime}$ in $[1$, p. 95] and Theorem 2.1 in [2], namely by constructing a representation $\varrho$ of $M \cap(F R)^{\perp}$ into $\Lambda \cap(F R)^{\perp}$ satisfying suitable congruence conditions at the primes dividing the discriminant of one of $\Lambda, R$ and glueing it to the given $\sigma$, the congruence conditions being chosen so that $\varrho \perp \sigma$ actually maps $M$ into $\Lambda$. 
Notice that the condition of bounded imprimitivity implies that the index of $M \cap(F R)^{\perp}$ in the orthogonal projection $\pi(M)$ of $M$ onto $(F R)^{\perp}$ is bounded independently of $M$ (for an integral primitive sublattice this index is bounded by the index of $R$ in its dual lattice $R^{\#}$ ). Notice (for comparison with Theorem 2.1 of [2]) that the boundedness of this index also implies that the quotient of the minimum of $M \cap(F R)^{\perp}$ and the minimum of $\pi(M)$ is bounded by some constant independent of $M$, so it is relevant only for the size of the constant $C$ which of these minima one considers.

Results of Kitaoka allow one to lift the condition on bounded imprimitivity in some cases with $n<2 m+3$ too:

Corollary 10. Let $F=\mathbb{Q}$, let $(V, Q), \Lambda$ be as before and fix a prime $q$ and $j \in \mathbb{N}$.

(i) Let $m \geq 6$ and $n=\operatorname{dim}(V) \geq 2 m$. Then there exists a constant $C:=C(\Lambda, j, q)$ such that $\Lambda$ represents all $\mathbb{Z}$-lattices $M$ of rank $m$ which are represented by $\Lambda$ locally everywhere, have minimum $\geq C$ and satisfy $\operatorname{ord}_{q}(\operatorname{disc}(M)) \leq j$.

(ii) Let $m \geq 3$ and $n=\operatorname{dim}(V) \geq 2 m+1$. Then there exists a constant $C:=C(\Lambda, j, q)$ such that $\Lambda$ represents all $\mathbb{Z}$-lattices $M$ of rank $m$ which are represented by $\Lambda$ locally everywhere, have minimum $\geq C$, satisfy $\operatorname{ord}_{q}(\operatorname{disc}(M)) \leq j$, and in the case $m=3$ are such that the orthogonal complement of $M_{q}$ in $\Lambda_{q}$ is isotropic.

(iii) Let $m=2$ and $n=\operatorname{dim}(V) \geq 6$. Then there exists a constant $C:=$ $C(\Lambda, j, q)$ such that $\Lambda$ represents all $\mathbb{Z}$-lattices $M$ of rank $m$ which are represented by $\Lambda$ locally everywhere, have minimum $\geq C$, satisfy $\operatorname{ord}_{q}(\operatorname{disc}(M)) \leq j$, and are such that the orthogonal complement of $M_{q}$ in $\Lambda_{q}$ is isotropic.

(iv) Let $M_{0}$ be a positive definite $\mathbb{Z}$-lattice of rank $m \leq n-3$ with Gram matrix $T_{0}$. Let $S$ be a finite set of primes with $q \in S$ such that:

(a) $\Lambda_{p}$ and $M_{p}$ are unimodular for all primes $p \notin S$ and for $p=q$,

(b) each isometry class in the genus of $\Lambda$ has a representative $\Lambda^{\prime}$ on $V$ such that $\Lambda_{p}^{\prime}=\Lambda_{p}$ for all primes $p \notin S$.

Then there exists a constant $C:=C\left(\Lambda, T_{0}, S\right)$ such that for all sufficiently large integers $a \in \mathbb{Z}$ which are not divisible by a prime in $S$, the $\mathbb{Z}$-lattice $M$ with Gram matrix a $T_{0}$ is represented by $\Lambda$ if it is represented by all completions $\Lambda_{p}$.

Proof. We notice first that the conditions in the various cases imply that the orthogonal complement of $M_{q}$ in $\Lambda_{q}$ is isotropic.

In [8-11] Kitaoka proved that the following condition $\mathbf{R}_{m, n}$ is satisfied for the values $m, n$ given in the assertions (with $M$ restricted to scalings of a fixed $M_{0}$ as described above in case (iv)): 
$\left(\mathbf{R}_{m, n}\right)$ For any $C_{1}>0$ there exists a $C_{2}>0$ such that the following is true: If $M$ is represented locally everywhere by $\Lambda$ and satisfies $\mu(M) \geq C_{2}$, then there exists a lattice $M^{\prime} \supseteq M$ of rank $m$ with minimum $\mu\left(M^{\prime}\right) \geq C_{1}$ which is primitively represented by $\Lambda$ locally everywhere.

Since for $C_{1}$ large enough the theorem asserts that $M^{\prime}$ is globally primitively represented by $\Lambda$, we obtain the representation of $M$ by $\Lambda$ if $C$ in the corollary is $\geq C_{2}$.

For the convenience of more matrix oriented readers we conclude by giving a matrix version (not in "geometrischer Einkleidung", see [16]) of the main result in the case $F=\mathbb{Q}$ :

TheOrem 11. Let $S \in M_{n}^{\mathrm{sym}}(\mathbb{Z})$ be a positive definite integral symmetric $n \times n$-matrix, and fix a prime $q$ and positive integers $j, c$. Then there is a constant $C$ such that a positive definite matrix $T \in M_{m}^{\text {sym }}(\mathbb{Z})$ with $m \leq n-3$ is represented by $S$ (i.e., $T={ }^{t} X S X$ with $X \in M_{n m}(\mathbb{Z})$ ) provided it satisfies:

(i) For each prime $p$ there exists a matrix $X_{p} \in M_{n m}\left(\mathbb{Z}_{p}\right)$ with ${ }^{t} X_{p} S X_{p}$ $=T$ such that the elementary divisors of $X$ divide $c$ and the equations ${ }^{t} X_{q} S \mathbf{y}=\mathbf{0}$ and ${ }^{t} \mathbf{y} S \mathbf{y}=0$ have a nontrivial common solution $\mathbf{y} \in \mathbb{Z}_{q}^{n}$.

(ii) $q^{j} \nmid \operatorname{det}(T)$.

(iii) $\min \left\{{ }^{t} \mathbf{y} T \mathbf{y} \mid \mathbf{0} \neq \mathbf{y} \in \mathbb{Z}^{m}\right\}>C$.

The matrix $X$ may be chosen to have elementary divisors dividing $c$.

\section{References}

[1] S. Böcherer and S. Raghavan, On Fourier coefficients of Siegel modular forms, J. Reine Angew. Math. 384 (1988), 80-101.

[2] W. K. Chan, B. M. Kim, M.-H. Kim and B.-K. Oh, Extensions of representations of integral quadratic forms, Ramanujan J. 17 (2008), 145-153.

[3] M. Eichler, Quadratische Formen und orthogonale Gruppen, 2nd ed., Springer, 1974.

[4] —, Die Ähnlichkeitsklassen indefiniter Gitter, Math. Z. 55 (1952), 216-252.

[5] J. Ellenberg and A. Venkatesh, Local-global principles for representations of quadratic forms, Invent. Math. 171 (2008), 257-279.

[6] J. S. Hsia, Representations by spinor genera, Pacific J. Math. 63 (1976), 147-152.

[7] J. S. Hsia, Y. Kitaoka and M. Kneser, Representations of positive definite quadratic forms, J. Reine Angew. Math. 301 (1978), 132-141.

[8] Y. Kitaoka, Local densities of quadratic forms, in: Investigations in Number Theory, Adv. Stud. Pure Math. 13, Academic Press, Boston, MA, 1988, 433-460.

[9] —, Some remarks on representations of positive definite quadratic forms, Nagoya Math. J. 115 (1989), 23-41.

[10] - , The minimum and the primitive representation of positive definite quadratic forms, ibid. 133 (1994), 127-153. 
[11] Y. Kitaoka, The minimum and the primitive representation of positive definite quadratic forms. II, ibid. 141 (1996), 1-27.

[12] —, Arithmetic of Quadratic Forms, Cambridge Tracts in Math. 106, Cambridge Univ. Press, Cambridge, 1993.

[13] M. Kneser, Darstellungsmaße indefiniter quadratischer Formen, Math. Z. 77 (1961), 188-194.

[14] -, Quadratische Formen, Springer, 2002.

[15] O. T. O'Meara, Introduction to Quadratic Forms, Springer, 1973.

[16] C. L. Siegel, Vorwort "Zur Reduktionstheorie quadratischer Formen", in: Gesammelte Abhandlungen IV, Springer, 1979, 329-330.

Fachrichtung 6.1 Mathematik

Universität des Saarlandes (Geb. E2.4)

Postfach 151150

66041 Saarbrücken, Germany

E-mail: schulzep@math.uni-sb.de

Received on 12.6.2008

and in revised form on 30.12.2008 